\title{
X-RAY AND ELECTRON-OPTICAL CHARACTERIZATION OF ZnSe(Co) CRYSTAL WITH NATURAL FACE
}

\author{
J. Auleytner ${ }^{a}$, J. Domagala ${ }^{a}$, Z. GolaCki ${ }^{a}$, M. PAWlowska $^{b}$, J. Pelka $^{a}$ \\ AND K. REGIŃSKI ${ }^{a}$ \\ a Institute of Physics, Polish Academy of Sciences \\ Al. Lotników 32/46, 02-668 Warszawa, Poland \\ ${ }^{b}$ Institute of Electronic Materials Technology, CEMI \\ Wólczyńska 133, 01-919 Warszawa, Poland
}

(Received December 11, 1992; revised version April 14, 1993)

\begin{abstract}
Using complementary X-ray and electron-optical methods, a $\mathrm{ZnSe}(\mathrm{Co})$ crystal with natural face was investigated. X-ray diffraction methods such as double-crystal X-ray reflection topography, double-crystal diffractometry for rocking curve measurements, precise lattice constant measurements by the Bond technique were used for crystal structure characterization and X-ray fluorescence method for studies of chemical composition along the crystal. The scanning electron microscopic image of the crystal surface and reflection diffraction of the high-energy electrons enriched the crystal structure characterization. It was shown that $\mathrm{X}$-ray characterization and reflection high-energy electron diffraction can be regarded as very important complementary tools for non-destructive investigation of the $\mathrm{ZnSe}(\mathrm{Co})$ crystal surface layers.
\end{abstract}

PACS numbers: $61.10 .-\mathrm{i}, 61.14 . \mathrm{Hg}, 61.16 . \mathrm{Bg}$

\section{Introduction}

The aim of the presented paper is to show what information can be obtained on the state of perfection of the natural faces of a crystal by using complementary $\mathrm{X}$-ray and electron-optical methods. An exact characterization of natural faces of a crystal is very important for surface properties research, for the choice of the proper condition of crystal growth and for the choice of an appropriate substrate to obtain more perfect epitaxial layers. The natural crystal faces can be regarded, in some cases, as very good substrates for molecular beam epitaxy (MBE) technique. In this work various $\mathrm{X}$-ray diffraction methods applied for the structure research were enriched by scanning electron microscopic (SEM) imaging of the crystal surface 
using secondary and backscattered electrons and by electron diffraction study using reflection high-energy electron diffraction (RIIEED) method. The X-ray methods are very useful for investigation of not only small-size crystals but the large ones as well. The information depth of $\mathrm{X}$-rays depends on their wavelength and on the a verage atomic number $Z$ of investigated sample; it is of order of $10^{3} \mathrm{~nm}$. The information depth of secondary electrons used for surface study is about $10 \mathrm{~nm}$. The information depth of backscattered electrons is of order of $10^{2} \mathrm{~nm}$ [1]. The information depth for RHEED at electron energy of $50 \mathrm{keV}$ is less than $10 \mathrm{~nm}$. Using the above mentioned methods, a state of perfection of the natural (111) facc of the Co doped ZnSe crystal obtained by chemical vapour transport was investigated.

\section{Single crystal growth}

Single crystals of $\mathrm{Zn}_{1-x} \mathrm{Co}_{x}$ Se where $x \approx 0.05$ were grown by chemical vapour transport method using iodine as the transporting agent. The starting materials were $\mathrm{ZnSe}$ powder (luminescence grade), Co $99.99 \%$ and Se $99.999 \%$ purity. First, we synthesized CoSe using stoichiometric weights of cobalt and selenium. Next, the CoSe boule was powdered and appropriate amount (about $15 \mathrm{~g}$ ) of $\mathrm{ZnSe}, \mathrm{CoSe}$ and iodine $\left(5 \mathrm{mg} / \mathrm{cm}^{3}\right)$ was introduced into silica tube with a conical tip.

The typical dimensions of the ampoules were: inner diameter $20 \mathrm{~mm}$ and length $100 \mathrm{~mm}$. The tubes were evacuated to $10^{-5} \mathrm{Tr}$, sealed off and placed into the horizontal furnace. The temperature conditions were rather typical of growing $\mathrm{ZnSc}$ doped crystals [2, 3].

The charge zone was maintained at $920^{\circ} \mathrm{C}$. The transport process was carried out for two weeks and the typical crystal of size $8 \times 8 \times 5 \mathrm{~mm}^{3}$ was grown in the colder part of the ampoule.

\section{Methods of X-ray characterization of the crystal face}

For characterization of the real structure of the crystal natural face the following methods were applied:

- X-ray reflection topography using the double-crystal traverse topography,

- double-crystal diffractometry for rocking curve measurements,

- precise lattice constant measurements by the Bond technique [4] - X-ray fluorescence for studies of chemical composition measured along the crystal (resolution about $0.5 \mathrm{~mm}$ ) [5],

- electron probe microanalysis using $\mathrm{X}$-ray emission of characteristic radiation due to the electron excitation of impurity atoms $[6,7]$ for the studies of local chemical composition (In this case the analyzing volume is in the order of several cubic microns.)

- X-ray standing wave method for qualitative estimation of the Co-atom localization in $\mathrm{ZnSe}$ crystal lattice [8].

The X-ray double-crystal spectrometer, constructed in the Central Laboratory of X-ray and Electron Microscope Research of the Institute of Physics (Polish Academy of Sciences) was set up in parallel $(+-)$ position. Since it is extremely 
difficult to find the $\mathrm{ZnSe}$ reference crystal without any structural defects, we used an Si single crystal of (111) orientation as the first monochromator for the X-ray beam. This monochromator was cut off from the bulk with asymmetry parameter equal to 0.1. Such an experimental setup (Si lattice constant $a=5.430995 \AA$, $\mathrm{ZnSe}(\mathrm{Co}) a=5.6691 \pm 2 \times 10^{-5} \AA$ ) was slightly dispersive. The half-width of the rocking curve of the $\mathrm{Si}$ reference crystal was $3.6 \mathrm{sec}$ of arc for (111) reflection. The Mo $K_{\alpha_{1}}$ radiation was eliminated by a slit placed in front of the investigated sample. The values of the half-width of $\mathrm{ZnSe}(\mathrm{Co})$ rocking curve varied from $9.5 \mathrm{sec}$ to 16 sec of arc depending on the investigated area of the crystal (see Fig. 1).
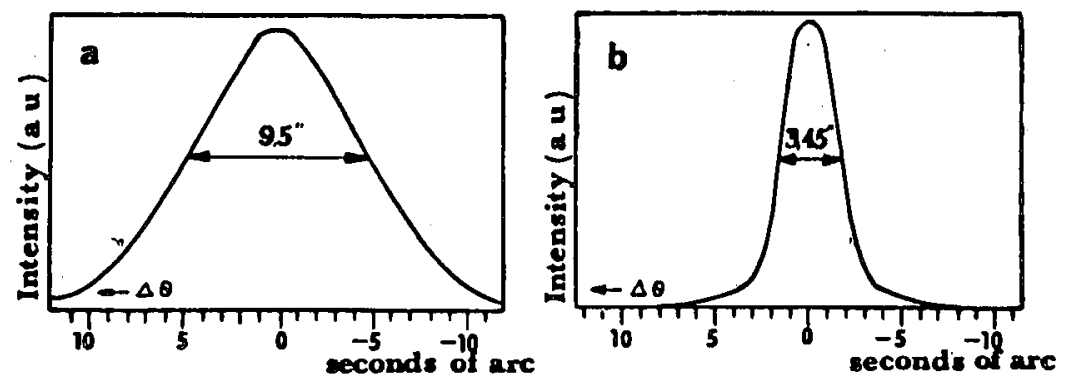

Fig. 1. (a) The typical rocking curve of the investigated $\mathrm{ZnSe}(\mathrm{Co})$ crystal (reflection (111)), $I$ - intensity, half-width of the rocking curve (in seconds of arc); (b) the rocking curve of Si reference crystal (reflection (111)).

For (111)-ZnSe(Co) reflection the theoretical half-width was calculated assuming strictly parallel incident beam using the following formula:

$$
\Delta \theta_{\mathbf{B}}=2 \sqrt{\frac{\gamma_{0}}{\left|\gamma_{h}\right|}} \frac{\left(\chi_{h}^{\mathrm{r}} \chi_{h}^{\mathrm{r}}-\chi_{h}^{\mathrm{i}} \chi_{h}^{\mathrm{i}}\right)^{1 / 2}}{\sin \left(2 \theta_{\mathrm{B}}\right)}
$$

We denote: $\gamma_{0}=\sin \left(\theta_{\mathrm{B}}-\alpha\right)$ for incident wave, $\gamma_{h}=\sin \left(\theta_{\mathrm{B}}+\alpha\right)$ for diffracted wave, $\alpha$ - angle between reflecting plane and crystal surface,

$$
\chi_{h}^{\mathrm{r}, \mathrm{i}}=r_{\mathrm{e}} \frac{\lambda^{2}}{\pi V} F_{h}^{\mathrm{r}, \mathrm{i}}
$$

where $r_{\mathrm{e}}$ - radius of an electron, $V$ - volume of the unit cell, $F_{h}^{\mathrm{r}, \mathrm{i}}-$ the real (r) and imaginary (i) part of the structure factor. For (111) $\mathrm{ZnSe}(\mathrm{Co})$ reflection $\Delta \theta$ is equal to $7.8 \mathrm{sec}$ of arc as calculated from the above formula for Mo $K_{\alpha_{1}}$ radiation.

The main reason of the differences in measured and calculated half-widths of the rocking curves is the imperfect structure of the investigated crystals. The existence of such imperfection was confirmed by scanning reflection topography.

Using the monochromatized X-ray beam and the simultaneous scanning of crystal and film, the topographs of the $\mathrm{ZnSe}(\mathrm{Co})$ crystals surface layer were obtained. The topographs revealed the number of singular extended structural and surface defects: scratches, cavities, growth dislocations and strains (see Fig. 5b).

To estimate the concentration deviation of $\mathrm{Zn}, \mathrm{Se}$, and Co atoms at the natural face (111) the X-ray fluorescence spectrometer with a semiconductor detector 
(EDAX) was used (see Fig. 2). Ouly small deviations from stoichiometry were observed (see Fig. 3) which means that Co-defects distribution is rather homogeneous. The electron probe microanalysis also did not show any precipitation of Co.

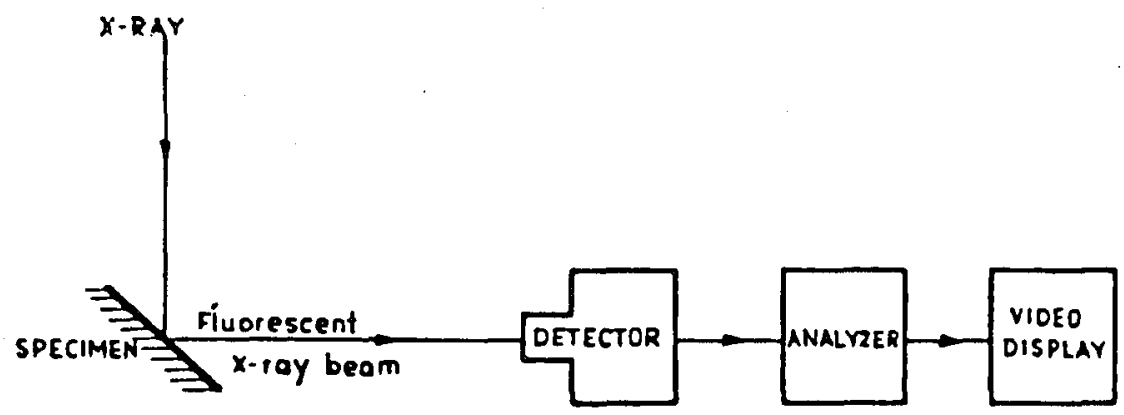

Fig. 2. The principle of the method for estimation of concentration of $\mathrm{Zn}, \mathrm{Co}$, and $\mathrm{Se}$ atoms along the natural face (111) by X-ray fluorescence spectrometer with a semiconductor detector (EDAX).

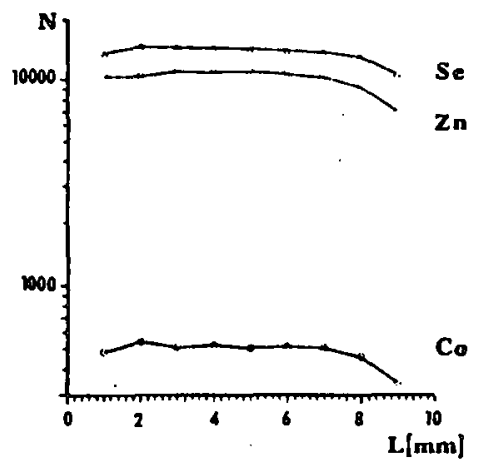

Fig. 3. Signals of fluorescence from $\mathrm{Zn}, \mathrm{Co}$, Se atoms measured along the natural face of $\mathrm{ZnSe}(\mathrm{Co}) . L-$ the length of the crystal, $N-$ number of counts.

To find the Co atoms position in the crystal lattice X-ray standing wave (XSW) method was applied. This method, in general, consists in simultaneous measurements of the rocking curve of the investigated crystal and the fluorescence radiation excited by the X-ray wave field. The XSW measurements need a double crystal spectrometer with two coincident detection systems: one scintillation counter for rocking curve measurements, the other for fluorescence analysis - solid state detector [9].

Figure 4 shows the measured rocking curve of $\mathrm{ZnSe}(\mathrm{Co})$ crystal and fluorescence yield angular dependence of $\mathrm{Se} K_{\alpha}, \mathrm{Zn} K_{\alpha}$ and Co $K_{\alpha}$ spectral lines. Though 

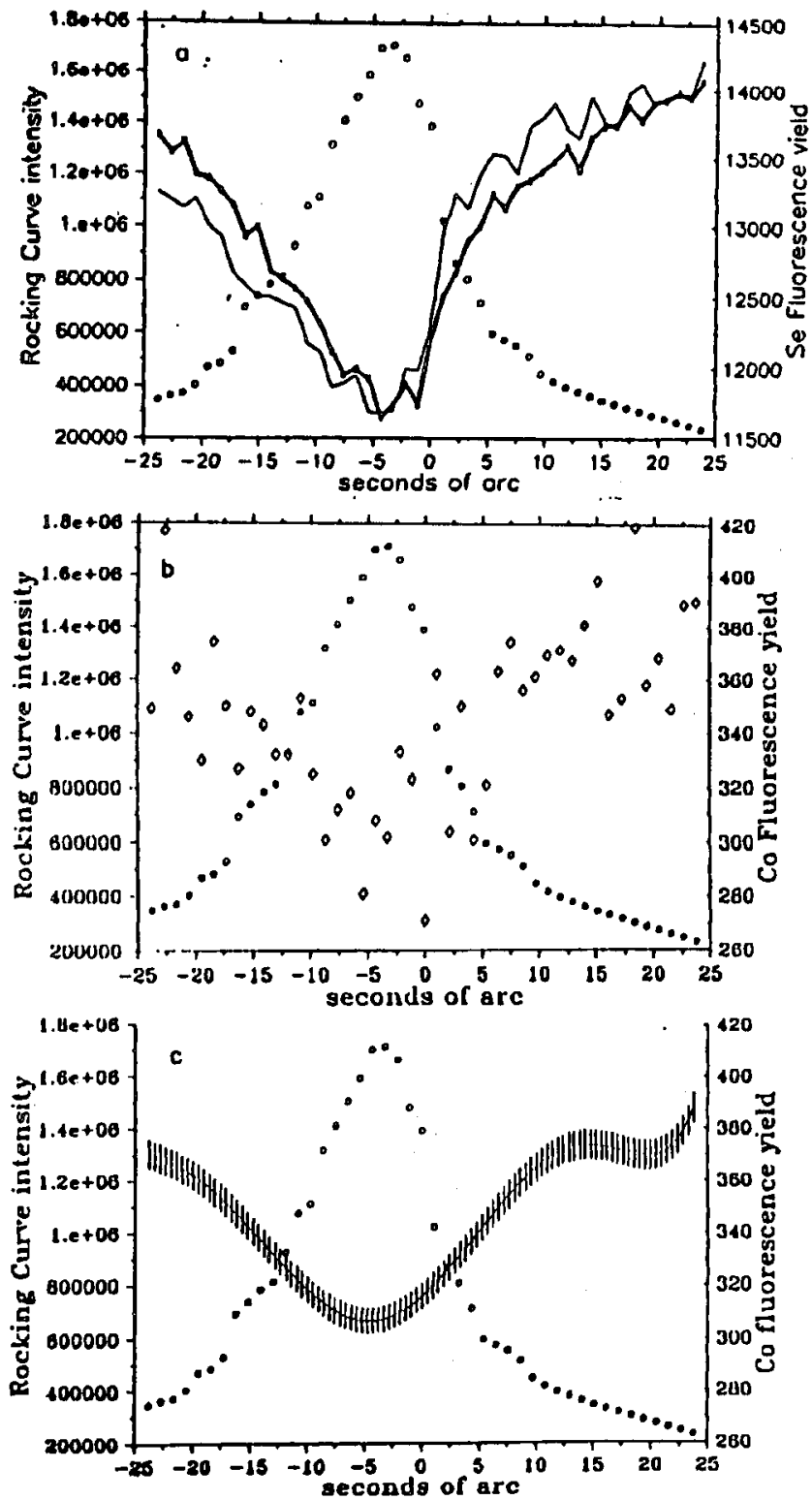

Fig. 4. The measured rocking curve of $\mathrm{ZnSe}(\mathrm{Co})$ crystal and $\mathrm{X}$-ray fluorescence angular dependence of the intensity of $\mathrm{Zn} K_{\alpha}$, Co $K_{\alpha}$ and Se $K_{\alpha}$ lines: (a) circles - rocking curve for $\mathrm{ZnSe}(\mathrm{Co})$, solid lines - fuorescence yields (thick - $\mathrm{Zn}$, thin - Se), (b) circles - rocking curve for $\mathrm{ZnSe}(\mathrm{Co})$, rombs - fluorescence yield for $\mathrm{Co}$, (c) circles rocking curve for $\mathrm{ZnSe}(\mathrm{Co})$, vertical lines approximated by polynomial curve for Co. Polynomial: $y=315.941+3.74649^{\prime \prime} x+0.335036^{\prime \prime} x^{2}-0.0159653^{\prime \prime} x^{3}-0.000837565^{\prime \prime} x^{4}$ $+\left(1.76923 \times 10^{-5}\right)^{\prime \prime} x^{5}+\left(7.82085 \times 10^{-7}\right)^{\prime \prime} x^{6}$. 
the investigated crystal cannot be considered as a perfect one (measured rocking curve much broader than the calculated one), the effect typical of the bulk perfect crystal as the decreasing fluorescence yield near the middle of the rocking curve is clearly visible. The minimum of Co curve coincides qualitatively well with the maximum of the rocking curve and minimum of the $\mathrm{Zn}$ fluorescence curve. Consequently, we can assume that the Co atoms are located mainly in the position of $\mathrm{Zn}$ atoms, which means substitutionally [10]. Due to the fact that Co concentration was relatively low the measured Co-fluorescence signals have a very pure statistic. Figure 4c shows the approximated curve which better defines the position of the minimum. This angular dependence of fluorescence yield can be approximated by the sixth-order polynomial. Extended defects in the small angular grain boundary and dislocations have not so large effect on the character of the angular fluorescence yield distribution, however, some perturbations of fluorescence yield form can be noticed $[10,8]$.

\section{Electron-optical characterization}

\subsection{Scanning electron microscopic (SEM) characterization of cryslal face}

Figure 5a shows a SEM image of the investigated natural face using secondary electrons. The magnification is small $(10 x)$, therefore the shape of the whole crystal is visible. The topography shows that the natural face is smooth. Only few microdefects are visible as bright spots. Figure $5 \mathrm{~b}$ shows, for comparison, the X-ray reflection topogramme of the same face. Dislocation lines, the other type
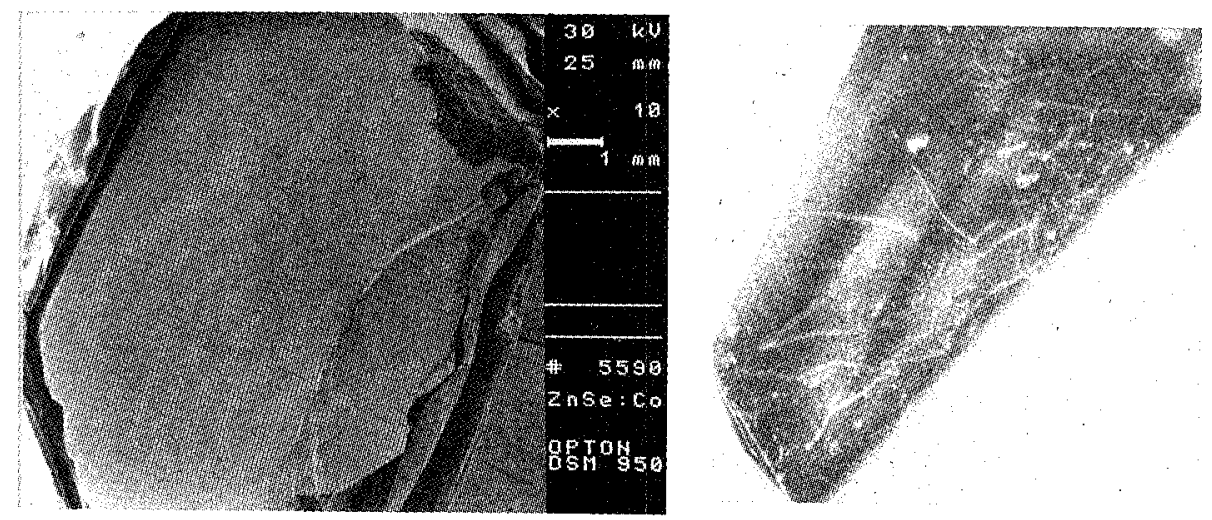

Fig. 5. (a) The SEM image of the investigated $\mathrm{ZnSe}(\mathrm{Co})$ natural crystal face (111) using secondary electrons (magnification 10x), (b) X-ray topogramme for comparison (nearly the same magnification).

of defects, and strain non-homogeneities are visible. Figure 6 shows the typical local image obtained by backscattered electrons (200x magnification). Microdefects of relatively low density are visible. In backscattered electron image the contrast depends mainly on atomic number. Therefore, we can suppose that the bright points correspond to Co or Co-rich precipitations with small density. 


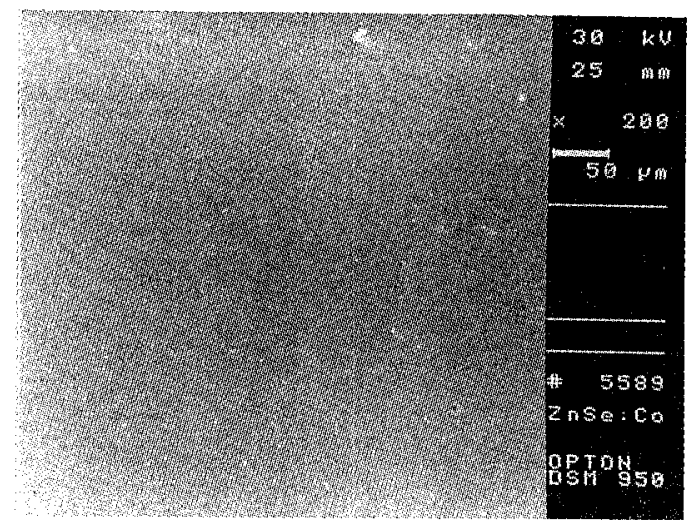

Fig. 6. The SEM local image of $\mathrm{ZnSe}(\mathrm{Co})$ natural crystal face. The bright points correspond to $\mathrm{Co}$ or Co-rich precipitations with small density.

\subsection{Sludy by RHEED}

The reflection high-energy electron diffraction (RHEED) technique was applied to characterize the crystal structure of the surface layer of the natural (111) face of $\mathrm{ZnSe}(\mathrm{Co})$ crystal. The used apparatus was an EMR-102 diffractometer supplied with an electron gun working in the energy range from 10 to $100 \mathrm{keV}$. The system has a precise manipulator of samples which enables to change the incidence angle $\theta$ of the electron beam within a very large range and the azimuth angle in the range $(0,2 \pi)$.

Because of irregular shape of the sample and some roughness (in microscopic sense) of the (111) surface it was not possible to observe the diffraction picture at all azimuths. The azimuths of the incident beam were adjusted so as to obtain satisfactory quality of the diffraction pattern.

Figure 7 shows a RHEED pattern taken in a [11̄̄] azimuth. Small deflection $\delta$ from the exact azimuth was applied to get the intersection of the Kikuchi lines with the spot pattern in order to enhance its intensity. The angle of incidence is $\theta=2.5^{\circ}$ and the energy of electron beam is $50 \mathrm{keV}$. In the case of diffraction conditions mentioned above the RHEED pattern refers to layers situated at depths of a few nanometers, therefore we observe the structure of the surface layer from the su:face to these levels. In Fig. 7 one can see a system of sharp and intense Bragg reflections and well-developed system of the Kikuchi lines. Such a picture is characteristic of good monocrystal structure. In our case this pattern evidences the perfection of the crystal structure of the surface layer. No admixture of other crystalline or polycrystalline phases is seen. However, observations in other azimuths show that the surface is not flat in the microscopic sense.

These conclusions are confirmed by the RHEED pattern shown in Fig. 8 . This pattern is taken in a [110] azimuth at the incidence angle $\theta=2.5^{\circ}$ and the energy of electron beam $75 \mathrm{keV}$. In the picture, besides the Bragg reflections and the Kikuchi lines intense Kikuchi bands are visible. Moreover, a weak specular 

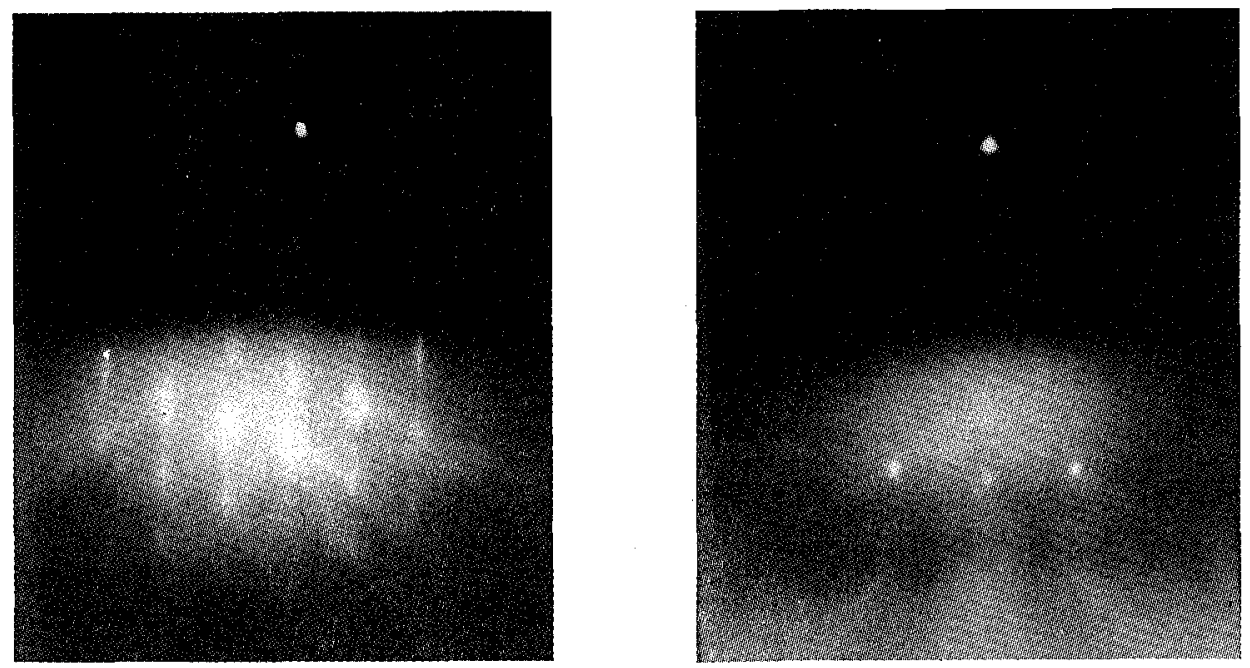

Fig. 7. RHEED pattern from (111) surface of $\mathrm{ZnSe}(\mathrm{Co})$ crystal, $[11 \overline{2}]+\delta$ azimuth, energy of electron beam $50 \mathrm{keV}$, incidence angle $\theta=2.5^{\circ}$.

Fig. 8. RHEED pattern from (111) surface of $\mathrm{ZnSe}(\mathrm{Co})$ crystal, [1ī0] azimuth, energy of electron beam $75 \mathrm{keV}$, incidence angle $\theta=2.5^{\circ}$.

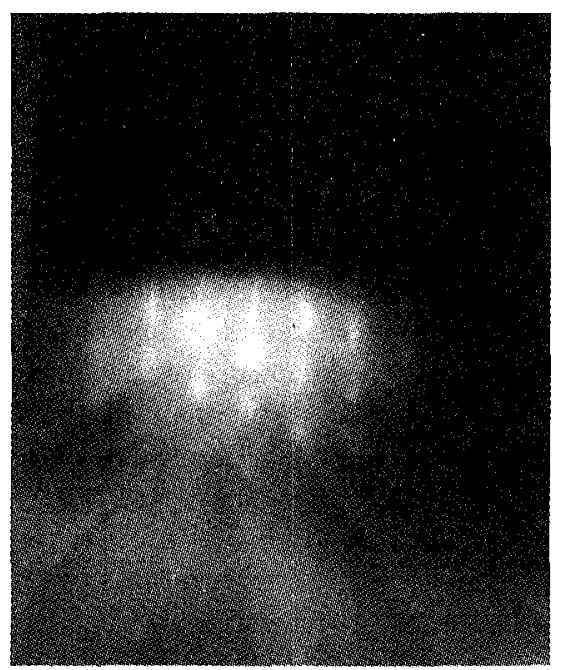

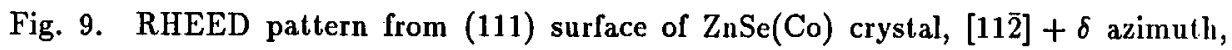
energy of electron beam $75 \mathrm{keV}$, incidence angle $\theta=2^{\circ}$. 
spot is observed near the point of intersection of the oblique Kikuchi lines. These elements of diffraction picture additionally confirm the perfection of the crystal structure of the surface layer.

The third pattern (Fig. 9) is taken in the same azimuth as Fig. 7, but at the incidence angle $\theta=2^{\circ}$ and the electron beam energy $75 \mathrm{keV}$. As we can see, the increase in the penetration depth does not result in change of the characteristic features of diffraction pattern. It evidences the homogeneity of the surface layer and confirms once more its good quality.

The results obtained by the RHEED technique correlate well with those obtained by SEM. It should be emphasized that, comparing with X-ray characterization, in the case of RHEED the thickness of the analysed layer is at least $10^{4}$ times smaller. It is obvious that the structure properties of the very thin surface layer can differ from those of a bulk. Thus X-ray characterization and RHEED can be regarded as complementary tools for non-destructive characterization of the $\mathrm{ZnSe}(\mathrm{Co})$ crystal.

\section{Conclusion}

For proper characterization of the state of perfection of the single crystal surface layer it is necessary to use different X-ray and electron-optical methods as complementary techniques. The physical phenomena deciding on image formation determine the information depth, and, on the other hand, the revealed images depend on the character of crystal lattice perturbations. The information depth in the case of X-ray diffraction methods applied in this work is about $3 \times 10^{3} \mathrm{~nm}$, for backscattered electrons about $10^{2} \mathrm{~nm}$, for secondary ones $10 \mathrm{~nm}$ and for RHEED less than $10 \mathrm{~nm}$. It is particularly clearly visible if we compare the secondary electron image of $\mathrm{ZnSe}(\mathrm{Co})$ natural face with the $\mathrm{X}$-ray traverse topogramme of the same crystal. In the latter case the defects and the lattice strain non-homogeneities from the depth of $10^{3} \mathrm{~nm}$, which were absent in the surface layer imaged by electron-optical methods, are revealed.

Therefore, one can conclude that the X-ray methods, the RHEED and SEM techniques can be regarded as very effective complementary tools for non-destructive characterization of the natural crystal faces.

\section{Acknowledgments}

The authors wish to thank Dr. P. Dłużewski and Dr. J. Bąk-Misiuk for discussion and some advice.

The technological part of this work was supported in part by the Commiltee for Scientific Research grant No. 223149102.

\section{References}

[1] L. Reiner, G. Pfefferkorn, in: Raster Electronmicroscopie, Eds. L. Reiner, G. Pfefferkorn, Springer-Verlag, Berlin 1973 p. 33.

[2] C.-M. Niu, R. Kershaw, K. Dwight, A. Wold, J. Solid State Chem. 85, 262 (1990). 
[3] W. Giriat, J.K. Furdyna, in: Semiconductors and Semimelals, Vol. 25, Diluled Magneto-Semiconductors, Eds. R.K. Willardson, A.L. Beer, Academic Press, New York 1988, p. 22.

[4] W.L. Bond, Acta Crystallogr: 13, 814 (1960).

[5] J. Auleytner, in: Proc. IVth Int. Summer School on Defects, Eds. T. Figielski, M. Jastrzębska, Z. Damm, PWN, Warszawa 1973, p. 158.

[6] J.B. Borowska, N.P. Iljin, Dokl. Akad. Nauk SSSR 106, 655 (1956).

[7] O. Brummer, J. Heydenreich, K.H. Krebs, in: Festkörperanalyse mit Elektronen, Jonen und Roentgenstrahlen, Eds. H. Bethge, J. Heyden reich, VEB Deutscher Verlag der Wissenschaften, Berlin 1980, p. 115.

[8] J. Pełka, J. Auleytner, Acta Phys. Pol. A 82, 163 (1992).

[9] J. Auleytner, J. Majewski, Z. Furmanik, Z. Golacki, Cryst. Res. Technology 25, 971 (1990).

[10] E.Kh. Mukhamedzhanov, Solid State Phenomena, Vol. 19, 20, Eds. M. Kittler, H. Richter, Trans Tech. Publications, Frankfurt (Oder) 1991, p. 477. 